\title{
Perfil epidemiológico dos acidentes por animais peçonhentos notificados no Estado de Minas Gerais durante o período de 2010-2015
}

\author{
Patrick Leonardo Nogueira da Silva \\ Professor da Escola Técnica de Saúde da Universidade Estadual de Montes Claros - UNIMONTES \\ Enfermeiro especialista em Saúde da Família e Enfermagem do Trabalho \\ Mestrando em Saúde, Sociedade e Ambiente pela Universidade Federal dos Vales do Jequitinhonha e Mucuri - \\ UFVJM \\ \patrick_mocesp70@hotmail.com
}

\section{Amanda de Andrade Costa}

Professora e Mestranda em Ciências da Saúde da Universidade Estadual de Montes Claros - UNIMONTES Servidora pública da Superintendência Regional de Saúde de Montes Claros - SRSMoc Enfermeira especialista em Políticas Públicas e Gestão de Saúde

Renata Fiúza Damasceno

Professora Mestre em Ciências da Saúde da Universidade Estadual de Montes Claros - UNIMONTES Servidora pública da Superintendência Regional de Saúde de Montes Claros - SRSMoc Enfermeira especialista em Políticas Públicas e Gestão de Saúde

\section{Ana Izabel de Oliveira Neta}

Enfermeira especialista em Saúde Coletiva Professora do curso de graduação em Enfermagem das Faculdades Santo Agostinho - FASA

Isabelle Ramalho Ferreira

Acadêmica do curso de Odontologia pela Universidade Estadual de Montes Claros - UNIMONTES

\section{Adélia Dayane Guimarães Fonseca}

Mestre e Doutoranda em Ciências da Saúde pela Universidade Estadual de Montes Claros - UNIMONTES Professora do curso de graduação em Enfermagem das Faculdades Santo Agostinho - FASA

\section{Resumo:}

Os acidentes por animais peçonhentos constituem grave problema de saúde pública, sendo por isso, considerados agravos tropicais negligenciados que acometem, na maior parte dos casos, populações de classe baixa residentes em áreas rurais. Os principais responsáveis por estes acidentes são aracnídeos e ofídios. 0 presente manuscrito objetiva identificar o perfil epidemiológico dos acidentes causados por animais peçonhentos notificados no Estado de Minas Gerais. Trata-se de estudo descritivo, exploratório, transversal com abordagem quantitativa, realizado no banco de dados de acesso público do Departamento de Informática do Sistema Único de Saúde. A amostra foi composta por 146.508 acidentes registrados durante o período de 2010-2015. Houve prevalência no ano de 2014, durante o mês de janeiro. Observou-se predominância do sexo masculino, entre 20-39 anos, sem registro de escolaridade, pardos e não aplicabilidade da condição gestacional. O acidente escorpiônico apresentou maior número de notificação. Em 48,8\% das vítimas, o tempo entre a picada e o atendimento foi inferior à uma hora. Quanto à classificação final e sua evolução, predominaram-se os acidentes leves e a cura, respectivamente. Portanto, os adultos jovens do sexo masculino apresentam maior vulnerabilidade aos acidentes, menor predisposição ao desenvolvimento sintomático e maior probabilidade de cura quando atendidos em tempo hábil. O escorpião apresenta-se como o principal vetor destes acidentes, bem como o principal causador de óbitos.

Palavras-chave: Animais venenosos, Picadas de escorpião, Mordeduras de serpentes, Sistemas de informação em saúde. 


\title{
Epidemiological profile of accidents by venomous animals reported in the State of Minas Gerais during the period 2010-2015
}

\begin{abstract}
:
Accidents involving venomous animals are a serious public health problem and are therefore considered to be neglected tropical diseases, which in most cases affect low-income populations living in rural areas. The main responsible for these accidents are arachnids and snakes. This manuscript aims to identify the epidemiological profile of accidents caused by venomous animals reported in the State of Minas Gerais. This was a descriptive, exploratory, cross-sectional study with a quantitative approach, carried out in the public access database of the Department of Information Technology of the Unified Health System. The sample consisted of 146,508 accidents recorded during the period between 2010 and 2015. There was prevalence in the year 2014, during the month of January. It was observed predominance of males, between 20-39 years old, with no schooling, brown and non-applicability of the gestational condition. The accident produced by scorpions had a higher number of notifications. In $48.8 \%$ of the victims, the time between the sting and the care was less than one hour. Regarding the final classification and its evolution, light accidents and cure were predominant, respectively. Therefore, young adult males are more vulnerable to accidents, less predisposed to symptomatic development, and more likely to be cured when seen in a timely manner. The scorpion is the main vector of these accidents, as well as the main cause of death.
\end{abstract}

Keywords: Animals poisonous, Scorpion stings, Snake bites, Health information systems.

\section{Perfil epidemiológico de los accidentes por animales venenosos notificados en el Estado de Minas Gerais durante el período de 2010-2015}

\section{Resumen:}

Los accidentes por animales venenosos constituyen un grave problema de salud pública, por lo que se consideran agravios tropicales descuidados que afectan en la mayoría de los casos a poblaciones de clase baja residentes en zonas rurales. Los principales responsables de estos accidentes son arácnidos y ofídicos. El presente manuscrito objetiva identificar el perfil epidemiológico de los accidentes causados por animales venenosos notificados en el Estado de Minas Gerais. Se trata de un estudio descriptivo, exploratorio, transversal con abordaje cuantitativo, realizado en el banco de datos de acceso público del Departamento de Informática del Sistema Único de Salud. La muestra fue compuesta por 146.508 accidentes registrados durante el período 2010-2015. Se produjo una prevalencia en el año 2014 durante el mes de enero. Se observó predominio del sexo masculino, entre 20-39 años, sin registro de escolaridad, pardos y no aplicabilidad de la condición de embarazada. El accidente escorpiônico presentó mayor número de notificación. En el 48,8\% de las víctimas, el tiempo entre la picadura y la atención fue inferior a una hora. En cuanto a la clasificación final y su evolución, predominaron los accidentes leves y la curación, respectivamente. Por lo tanto, los adultos jóvenes del sexo masculino presentan mayor vulnerabilidad a los accidentes, menor predisposición al desarrollo sintomático y mayor probabilidad de curación cuando son atendidos en tiempo hábil. El escorpión se presenta como el principal vector de estos accidentes, así como el principal causante de muertes.

Palabras clave: Animales venenosos, Picaduras de escorpión, Mordeduras de serpientes, Sistemas de información en salud.

\section{INTRODUÇÃO}

Os ofídios, aranhas e escorpiões são os principais animais peçonhentos de interesse em saúde pública, pois causam acidentes cuja gravidade varia de leve a grave (BRASIL, 2016). Os escorpiões, por sua vez, são os responsáveis por quase a metade das notificações 
registradas no Sistema de Informação de Agravos de Notificação (SINAN). Com o surgimento das chuvas, aumenta o risco de acidentes com animais peçonhentos, por exemplo, no ano de 2012, o índice pluviométrico trouxe aumento de quase $6 \%$ nas notificações de acidentes, especialmente com escorpiões. Assim, registraram-se entre novembro de 2012 e março de 2013, temporada de chuvas na maior parte do país, 71.217 acidentes e 144 óbitos. Em comparação com o mesmo período do ano anterior, houve um acréscimo de quase $6 \%$ no número de acidentes com estes animais e $9 \%$ em relação a óbitos. Neste período, foram notificados 67.197 casos e 132 óbitos. Em todo o ano de 2012, foram notificados 143.658 acidentes com 273 mortes (BRASIL, 2014).

O habitat natural modificado por ações antrópicas causa quebra na cadeia alimentar, acabando também com os abrigos. Com essa escassez, esses animais migram para as residências urbanas, lotes e terrenos baldios, áreas de construção, locais propícios ao desenvolvimento de artrópodes em decorrência do acúmulo de matéria orgânica, de modo a objetivar a busca por abrigo e alimento. Sendo assim, tornam-se vulneráveis a estes ataques as crianças, donas de casa e trabalhadores de construção civil, além de outros trabalhadores braçais (COSTA, 2011). Na realidade, esta relação pode ser dada por meio da exposição da população aos animais, uma vez que, com o aumento de chuvas os animais buscam abrigo, se aproximando mais das habitações humanas.

Das 86 espécies de escorpiões catalogadas no Brasil, apenas seis, pertencentes ao gênero Tityus, são causadoras de acidentes graves entre humanos, são elas, Tityus cambridgei e T. metuendus, encontrados na região amazônica; T. bahiensis, encontrado no Sudeste e no norte da região Sul, sendo inclusive a espécie que provoca mais acidentes no Estado de São Paulo; T. costatus, encontrado do Estado Minas Gerais ao do Rio Grande do Sul pela zona costeira coberta por Mata Atlântica; T. serrulatus, encontrado nos Estados da Bahia, Minas Gerais, São Paulo, Rio de Janeiro, Espírito Santo, Goiás e Distrito Federal, o escorpião responsável pelo maior número deacidentes graves e óbitos no Brasil; e T. stigmurus, encontrado no norte de Minas Gerais eem todos os Estados do Nordeste, com exceção do Maranhão (SILVA et al., 2005).

Acidentes causados por aranhas são comuns, porém a maioria não apresenta repercussão clínica e não são prevalentes quando comparados aos acidentes escorpiônicos. Os gêneros de importância em saúde pública no Brasil são: Loxosceles (aranha-marrom), 
Phoneutria (aranha-armadeira ou macaca) e Latrodectus (viúva-negra). Entre essas, a maior causadora de acidentes é a Loxosceles (BRASIL, 2001).

Já o acidente ofídico ou ofidismo é o quadro de envenenamento decorrente da inoculação da peçonha através das picadas das serpentes. No Brasil, as serpentes peçonhentas de interesse em saúde pública são representadas por quatro gêneros da Família Viperidae; serpentes do gênero Bothrops, vulgarmente denominadas de jararaca, jararacuçu, urutu, caiçaca e comboia, estão agrupadas em dois gêneros - Bothrops e Botrocophias; Crotalus (cascavel); Lachesis (surucucu-pico-de-jaca); Micrurus e Leptomicrurus (coral-verdadeira) (SANTANA; SUCHARA, 2015).

A seguir, o Quadro 1 relaciona várias espécies de animais peçonhentos que são de grande importância na saúde pública em decorrência do alto índice de acidentes causados pelos mesmos na qual foram distribuídos por regiões e Estados brasileiros. 
Quadro 1 - Relação das espécies de animais peçonhentos de importância na saúde pública distribuídos por regiões e Estados brasileiros.

\begin{tabular}{|c|c|c|c|c|}
\hline \multirow{2}{*}{ Regiões } & \multirow{2}{*}{ Estados } & \multicolumn{3}{|c|}{ Espécies de animais peçonhentos } \\
\hline & & Escorpionídeos & Ofídios & Aracnídeos \\
\hline \multirow{6}{*}{ Norte } & Acre & Tityus paraensis & $\begin{array}{l}\text { Bothrocophias hyoprora } \\
\text { Bothrops atrox } \\
\text { Bothrops bilineatus }\end{array}$ & Loxosceles amazonica \\
\hline & Amapá & Tityus paraensis & $\begin{array}{l}\text { Bothrops atrox } \\
\text { Bothrops bilineatus } \\
\text { Crotalus durissus } \\
\text { Leptomicrurus collaris }\end{array}$ & Loxosceles amazonica \\
\hline & Amazonas & $\begin{array}{l}\text { Tityus paraensis } \\
\text { Tityus cambridgei } \\
\text { Tityus metuendus }\end{array}$ & $\begin{array}{l}\text { Bothrocophias hyoprora } \\
\text { Bothrops atrox } \\
\text { Bothrops bilineatus } \\
\text { Bothrops brazili } \\
\text { Crotalus durissus } \\
\text { Lachesis muta } \\
\text { Leptomicrurus collaris }\end{array}$ & Loxosceles amazonica \\
\hline & Pará & Tityus paraensis & $\begin{array}{l}\text { Bothrocophias hyoprora } \\
\text { Bothrops atrox } \\
\text { Bothrops brazili } \\
\text { Crotalus durissus }\end{array}$ & $\begin{array}{l}\text { Loxosceles similis } \\
\text { Loxosceles amazônica }\end{array}$ \\
\hline & Rondônia & Tityus paraensis & $\begin{array}{l}\text { Bothrocophias hyoprora } \\
\text { Bothrocophias microphthalmus } \\
\text { Bothrops bilineatus } \\
\text { Bothrops brazili } \\
\text { Crotalus durissus }\end{array}$ & Loxosceles amazônica \\
\hline & Roraima & Tityus paraensis & $\begin{array}{l}\text { Bothrops atrox } \\
\text { Bothrops bilineatus } \\
\text { Crotalus durissus } \\
\text { Leptomicrurus collaris }\end{array}$ & Loxosceles amazônica \\
\hline
\end{tabular}




\begin{tabular}{|c|c|c|c|c|}
\hline & Tocantins & Tityus paraensis & $\begin{array}{l}\text { Bothrops atrox } \\
\text { Crotalus durissus } \\
\end{array}$ & Loxosceles amazônica \\
\hline \multirow{8}{*}{ Nordeste } & Alagoas & Tityus stigmurus & \begin{tabular}{|l|} 
Crotalus durissus \\
Lachesis muta \\
Micrurus ibiboboca
\end{tabular} & $\begin{array}{l}\text { Loxosceles amazônica } \\
\text { Phoneutria bahiensis } \\
\text { Phoneutria keyserlingi }\end{array}$ \\
\hline & Bahia & $\begin{array}{l}\text { Tityus serrulatus } \\
\text { Tityus bahiensis } \\
\text { Tityus stigmurus }\end{array}$ & $\begin{array}{l}\text { Bothrops bilineatus } \\
\text { Crotalus durissus } \\
\text { Lachesis muta } \\
\text { Micrurus brasiliensis } \\
\text { Micrurus ibiboboca }\end{array}$ & $\begin{array}{l}\text { Loxosceles amazônica } \\
\text { Phoneutria bahiensis } \\
\text { Phoneutria keyserlingi }\end{array}$ \\
\hline & Ceará & $\begin{array}{l}\text { Tityus serrulatus } \\
\text { Tityus stigmurus }\end{array}$ & $\begin{array}{l}\text { Crotalus durissus } \\
\text { Lachesis muta } \\
\text { Micrurus ibiboboca }\end{array}$ & $\begin{array}{l}\text { Loxosceles amazônica } \\
\text { Phoneutria bahiensis } \\
\text { Phoneutria keyserlingi }\end{array}$ \\
\hline & Maranhão & Tityus stigmurus & $\begin{array}{l}\text { Bothrops atrox } \\
\text { Crotalus durissus } \\
\text { Micrurus ibiboboca }\end{array}$ & Loxosceles amazônica \\
\hline & Paraíba & Tityus stigmurus & $\begin{array}{l}\text { Crotalus durissus } \\
\text { Lachesis muta } \\
\text { Micrurus ibiboboca } \\
\text { Micrurus potyguara }\end{array}$ & $\begin{array}{l}\text { Loxosceles amazônica } \\
\text { Loxosceles laeta } \\
\text { Phoneutria bahiensis } \\
\text { Phoneutria keyserlingi }\end{array}$ \\
\hline & Pernambuco & $\begin{array}{l}\text { Tityus serrulatus } \\
\text { Tityus stigmurus }\end{array}$ & $\begin{array}{l}\text { Crotalus durissus } \\
\text { Lachesis muta } \\
\text { Micrurus ibiboboca } \\
\text { Micrurus potyguara }\end{array}$ & $\begin{array}{l}\text { Loxosceles amazônica } \\
\text { Phoneutria bahiensis } \\
\text { Phoneutria keyserlingi }\end{array}$ \\
\hline & Piauí & $\begin{array}{l}\text { Tityus serrulatus } \\
\text { Tityus stigmurus }\end{array}$ & $\begin{array}{l}\text { Crotalus durissus } \\
\text { Lachesis muta } \\
\text { Micrurus ibiboboca }\end{array}$ & $\begin{array}{l}\text { Loxosceles amazônica } \\
\text { Phoneutria bahiensis } \\
\text { Phoneutria keyserlingi }\end{array}$ \\
\hline & Rio Grande do Norte & $\begin{array}{l}\text { Tityus serrulatus } \\
\text { Tityus stigmurus }\end{array}$ & $\begin{array}{l}\text { Crotalus durissus } \\
\text { Lachesis muta } \\
\text { Micrurus ibiboboca }\end{array}$ & $\begin{array}{l}\text { Loxosceles amazônica } \\
\text { Phoneutria bahiensis } \\
\text { Phoneutria keyserlingi }\end{array}$ \\
\hline
\end{tabular}


Perfil epidemiológico dos acidentes por animais peçonhentos notificados no Estado de Minas Gerais durante o período de 2010-2015

\begin{tabular}{|c|c|c|c|c|}
\hline & & & Micrurus potyguara & Latrodectus curacaviensis \\
\hline & Sergipe & $\begin{array}{l}\text { Tityus serrulatus } \\
\text { Tityus stigmurus }\end{array}$ & $\begin{array}{l}\text { Crotalus durissus } \\
\text { Lachesis muta } \\
\text { Micrurus ibiboboca }\end{array}$ & $\begin{array}{l}\text { Loxosceles amazônica } \\
\text { Phoneutria bahiensis } \\
\text { Phoneutria keyserlingi }\end{array}$ \\
\hline \multirow{4}{*}{ Centro-Oeste } & Distrito Federal & Tityus serrulatus & Crotalus durissus & \\
\hline & Goiás & $\begin{array}{l}\text { Tityus serrulatus } \\
\text { Tityus bahiensis }\end{array}$ & $\begin{array}{l}\text { Bothrops alternatus } \\
\text { Crotalus durissus } \\
\text { Lachesis muta } \\
\text { Micrurus brasiliensis }\end{array}$ & $\begin{array}{l}\text { Loxosceles intermédia } \\
\text { Phoneutria bahiensis } \\
\text { Phoneutria keyserlingi }\end{array}$ \\
\hline & Mato Grosso & Tityus bahiensis & $\begin{array}{l}\text { Bothrops atrox } \\
\text { Bothrops bilineatus } \\
\text { Bothrops brazili } \\
\text { Crotalus durissus } \\
\end{array}$ & \\
\hline & Mato Grosso do Sul & $\begin{array}{l}\text { Tityus serrulatus } \\
\text { Tityus bahiensis }\end{array}$ & $\begin{array}{l}\text { Bothrops alternatus } \\
\text { Crotalus durissus } \\
\text { Lachesis muta }\end{array}$ & $\begin{array}{l}\text { Loxosceles similis } \\
\text { Phoneutria bahiensis } \\
\text { Phoneutria keyserlingi }\end{array}$ \\
\hline \multirow{3}{*}{ Sudeste } & Espírito Santo & $\begin{array}{l}\text { Tityus serrulatus } \\
\text { Tityus bahiensis }\end{array}$ & $\begin{array}{l}\text { Bothrops bilineatus } \\
\text { Crotalus durissus } \\
\text { Lachesis muta }\end{array}$ & $\begin{array}{l}\text { Loxosceles intermédia } \\
\text { Loxosceles laeta } \\
\text { Phoneutria bahiensis } \\
\text { Phoneutria keyserlingi }\end{array}$ \\
\hline & Minas Gerais & $\begin{array}{l}\text { Tityus serrulatus } \\
\text { Tityus bahiensis } \\
\text { Tityus costatus }\end{array}$ & $\begin{array}{l}\text { Bothrops alternatus } \\
\text { Crotalus durissus } \\
\text { Lachesis muta }\end{array}$ & $\begin{array}{l}\text { Loxosceles similis } \\
\text { Loxosceles gaucho } \\
\text { Loxosceles intermédia } \\
\text { Loxosceles laeta } \\
\text { Loxosceles hirsuta } \\
\text { Phoneutria bahiensis } \\
\text { Phoneutria keyserlingi }\end{array}$ \\
\hline & Rio de Janeiro & $\begin{array}{l}\text { Tityus serrulatus } \\
\text { Tityus bahiensis }\end{array}$ & $\begin{array}{l}\text { Crotalus durissus } \\
\text { Lachesis muta }\end{array}$ & $\begin{array}{l}\text { Loxosceles intermédia } \\
\text { Loxosceles laeta } \\
\text { Loxosceles adelaida }\end{array}$ \\
\hline
\end{tabular}




\begin{tabular}{|c|c|c|c|c|}
\hline & & & & $\begin{array}{l}\text { Phoneutria bahiensis } \\
\text { Phoneutria keyserlingi } \\
\text { Latrodectus curacaviensis }\end{array}$ \\
\hline & São Paulo & $\begin{array}{l}\text { Tityus serrulatus } \\
\text { Tityus bahiensis }\end{array}$ & $\begin{array}{l}\text { Bothrops alcatraz } \\
\text { Bothrops alternatus } \\
\text { Crotalus durissus } \\
\text { Lachesis muta }\end{array}$ & $\begin{array}{l}\text { Loxosceles similis } \\
\text { Loxosceles gaucho } \\
\text { Loxosceles intermédia } \\
\text { Loxosceles laeta } \\
\text { Loxosceles adelaida } \\
\text { Loxosceles hirsuta } \\
\text { Phoneutria bahiensis } \\
\text { Phoneutria bahiensis } \\
\text { Phoneutria keyserlingi }\end{array}$ \\
\hline \multirow{3}{*}{ Sul } & Paraná & $\begin{array}{l}\text { Tityus serrulatus } \\
\text { Tityus bahiensis }\end{array}$ & $\begin{array}{l}\text { Bothrops alternatus } \\
\text { Crotalus durissus } \\
\text { Lachesis muta }\end{array}$ & $\begin{array}{l}\text { Loxosceles gaucho } \\
\text { Loxosceles intermédia } \\
\text { Loxosceles laeta } \\
\text { Loxosceles hirsuta } \\
\text { Phoneutria bahiensis } \\
\text { Phoneutria keyserlingi }\end{array}$ \\
\hline & Rio Grande do Sul & $\begin{array}{l}\text { Tityus bahiensis } \\
\text { Tityus costatus }\end{array}$ & $\begin{array}{l}\text { Bothrops alternatus } \\
\text { Crotalus durissus } \\
\text { Lachesis muta }\end{array}$ & $\begin{array}{l}\text { Loxosceles gaucho } \\
\text { Loxosceles intermédia } \\
\text { Loxosceles laeta } \\
\text { Loxosceles hirsuta } \\
\text { Phoneutria bahiensis } \\
\text { Phoneutria keyserlingi }\end{array}$ \\
\hline & Santa Catarina & $\begin{array}{l}\text { Tityus serrulatus } \\
\text { Tityus bahiensis }\end{array}$ & $\begin{array}{l}\text { Bothrops alternatus } \\
\text { Crotalus durissus } \\
\text { Lachesis muta }\end{array}$ & $\begin{array}{l}\text { Loxosceles gaucho } \\
\text { Loxosceles intermédia } \\
\text { Loxosceles laeta } \\
\text { Phoneutria bahiensis } \\
\text { Phoneutria keyserlingi }\end{array}$ \\
\hline
\end{tabular}

Fonte: Autoria própria, 2017. 
Diante de todo o território brasileiro, o Estado de Minas Gerais é a região que detém a maior parte das notificações de acidentes por animais peçonhentos o qual se justifica a realização deste estudo. Sendo assim, torna-se evidente a importância de uma investigação regional e local detalhada da incidência desses acidentes, assim como um levantamento das espécies que mais causam injúrias à população. O conhecimento desses casos e de sua evolução é fundamental para melhorias no atendimento médico e para o desenvolvimento de atividades de vigilância em saúde, objetivando o controle e prevenção desses acidentes. Portanto, este estudo objetiva identificar o perfil epidemiológico dos acidentes por animais peçonhentos notificados no Estado de Minas Gerais entre os de 2010 e 2015.

\section{MÉTODOS}

Trata-se de um estudo descritivo, exploratório, transversal, com abordagem quantitativa, realizado a partir da analise dos dados de acesso público do DATASUS. 0 universo da pesquisa açambarcou 146.508 registros de acidentes em todo Estado de Minas Gerais entre 2010 e 2015, dados com disponibilização online gratuita pelo site do DATASUS (http://datasus.saude.gov.br/informacoes-de-saude/tabnet) (BRASIL, 2017).

Foi utilizado um formulário estruturado de elaboração própria baseado na ficha do Sistema de Informação de Agravos de Notificação (SINAN) como instrumento de coleta de dados, que contemplou as seguintes variáveis: ano da notificação, mês da notificação, sexo, faixa etária, escolaridade, raça/cor, gestante, causador do acidente, causado por serpente (espécie), causado por aranha (espécie), tempo entre a picada e o atendimento, classificação final e evolução do caso. Os dados sobre as espécies de escorpiões causadoras de acidentes não foram abordadas em decorrência da ficha de notificação do SINAN não contemplá-las.

Os dados foram armazenados no Statistical Package for the Social Sciences (SPSS ${ }^{\circledast}$ ), versão 15.0, tabulados e apresentados em tabelas com frequências absolutas e relativas. As tabelas foram elaboradas através do programa estatístico Microsoft Excel ${ }^{\circledR}$, versão 2010- e o tratamento estatístico dos dados foi feito por meio de análise uni-variada. 
Por se tratar de dados de domínio público, não foi recomendada a aprovação do Comitê de Ética em Pesquisa (CEP).

\section{RESULTADOS}

Observou-se que os anos com maiores prevalências foram 2014 (n=29.868; 20,4\%), seguido do ano de 2013 ( $n=29.348 ; 20,0 \%)$. Já os meses de maior ocorrência dos acidentes foram, janeiro $(n=15.175 ; 10,3 \%)$, seguido de março $(n=14.431 ; 9,8 \%)$ e fevereiro $(n=13.601 ; 9,3 \%)$ (Tabela 1$)$.

Tabela 1 - Período de acometimento dos acidentes por animais peçonhentos no Estado de Minas Gerais (2010-2015), n=146.508.

\begin{tabular}{|c|c|c|}
\hline VARIÁVEIS & $\mathbf{n}$ & $\%$ \\
\hline \multicolumn{3}{|l|}{ Anos } \\
\hline 2015 & 19.355 & 13,2 \\
\hline 2014 & 29.868 & 20,4 \\
\hline 2013 & 29.348 & 20,0 \\
\hline 2012 & 25.456 & 17,4 \\
\hline 2011 & 22.465 & 15,3 \\
\hline 2010 & 20.016 & 13,7 \\
\hline \multicolumn{3}{|l|}{ Meses } \\
\hline Janeiro & 15.175 & 10,3 \\
\hline Fevereiro & 13.601 & 9,3 \\
\hline Março & 14.431 & 9,8 \\
\hline Abril & 13.199 & 9,0 \\
\hline Maio & 12.129 & 8,3 \\
\hline Junho & 10.931 & 7,5 \\
\hline Julho & 11.391 & 7,8 \\
\hline Agosto & 10.742 & 7,3 \\
\hline Setembro & 9.804 & 6,7 \\
\hline Outubro & 10.734 & 7,3 \\
\hline Novembro & 11.729 & 8,0 \\
\hline Dezembro & 12.642 & 8,7 \\
\hline
\end{tabular}

Fonte: DATASUS, 2017 
O perfil socioeconômico e clínico das vítimas é representado pela Tabela 2. Tem-se que 57,0\% eram do sexo masculino, adultos jovens com faixa etária entre 20-39 anos (31,7\%), a escolaridade foi ignorada em 91,7\% das notificações, 42,7\% declaram-se pardos e 69,2\% das vítimas apresentam não aplicabilidade da condição gestacional devido ao fato de que a maior parte dos notificados serem do sexo masculino.

Tabela 2 - Perfil socioeconômico e clínico das vítimas de acidentes por animais peçonhentos notificados no Estado de Minas Gerais (2010-2015), n=146.508.

\begin{tabular}{lcc}
\hline VARIÁVEIS & $\mathbf{n}$ & $\mathbf{\%}$ \\
\hline Sexo & & \\
Ignorado/Branco & 16 & 0,1 \\
Masculino & 83.586 & 57,0 \\
Feminino & 62.906 & 42,9 \\
& & \\
Faixa etária (anos) & & \\
Ignorado/Branco & 29 & 0,1 \\
$<1$ & 2.147 & 1,4 \\
$1-4$ & 6.557 & 4,5 \\
$5-9$ & 8.999 & 6,1 \\
$10-14$ & 10.325 & 7,0 \\
$15-19$ & 12.256 & 8,4 \\
$20-39$ & 46.470 & 31,7 \\
$40-59$ & 38.930 & 26,6 \\
$60-64$ & 6.904 & 4,7 \\
$65-69$ & 5.148 & 3,5 \\
$70-79$ & 6.454 & 4,4 \\
$\geq 80$ & 2.289 & 1,6
\end{tabular}

\section{Escolaridade}

Ignorado/Branco

$134.34491,7$

Nenhuma

$12.164 \quad 8,3$ 


\section{Cor/Raça}

\begin{tabular}{lcc} 
Ignorado/Branco & 21.158 & 14,4 \\
Branco & 48.666 & 33,1 \\
Preto & 12.040 & 8,2 \\
Amarelo & 1.590 & 1,0 \\
Pardo & 62.537 & 42,7 \\
Indígena & 517 & 0,6 \\
& & \\
Gestante & & \\
Ignorado/Branco & 9.490 & 6,7 \\
1ํtrimestre & 307 & 0,2 \\
2o trimestre & 441 & 0,3 \\
3ํㅡrimestre & 313 & 0,2 \\
Idade gestacional ignorada & 260 & 0,1 \\
Não & 34.237 & 23,3 \\
Não se aplica & 101.460 & 69,2 \\
\hline
\end{tabular}

Fonte: DATASUS, 2017.

Os escorpiões foram os responsáveis pela maioria dos acidentes acometendo 88.602 indivíduos (60,5\%). Neste estudo, destacam-se também os acidentes ofídicos (13,1\%) cujo principal foi o Botrópico (9,1\%). Os acidentes produzidos pelas aranhas apresentaram menor representatividade quando comparados aos acidentes produzidos pelos demais animais peçonhentos e a principal espécie envolvida foi a aranha-armadeira (Phoneutria nigriventer) $(1,9 \%)$.

O tempo decorrido entre a picada e o atendimento é crucial na recuperação da vítima e pode determinar a sua cura ou o seu óbito. Em 48,8\% dos casos, as vítimas receberam atendimento ambulatorial em tempo hábil inferior à uma hora. Houve predomínio de acidentes do tipo leve $(80,0 \%)$ com evolução de cura $(95,9 \%)$ (Tabela 3).

Destaca-se, com base nos dados apresentados, que na maioria dos acidentes não existe a correta identificação do agente causador, de modo a gerar obscuridade nos dados investigados. Dessa maneira, pode-se incluir maior relevância no sentido de, a correta 
identificação do animal causador do acidente, auxilia no tratamento, e também eleva a possibilidade de sucesso do mesmo.

Tabela 3 - Aspectos clínicos dos acidentes por animais peçonhentos ocorridos no Estado de Minas Gerais (2010-2015), $\mathrm{n}=146.508$.

\begin{tabular}{|c|c|c|}
\hline Variáveis & $\mathbf{n}$ & $\%$ \\
\hline \multicolumn{3}{|l|}{ Causador do acidente } \\
\hline Ignorado/Branco & 2.173 & 1,5 \\
\hline Escorpião & 88.602 & 60,5 \\
\hline Serpente & 19.272 & 13,1 \\
\hline Aranha & 15.993 & 10,9 \\
\hline Abelha & 9.044 & 6,2 \\
\hline Lagarta & 4.698 & 3,2 \\
\hline Outros & 6.726 & 4,6 \\
\hline \multicolumn{3}{|l|}{ Causado por serpente } \\
\hline Ignorado/Branco & 129.335 & 88,3 \\
\hline Botrópico & 13.264 & 9,1 \\
\hline Crotálico & 3.110 & 2,1 \\
\hline Micrurus corallinus & 116 & 0,09 \\
\hline Lachesis & 19 & 0,01 \\
\hline Não peçonhenta & 664 & 0,4 \\
\hline \multicolumn{3}{|l|}{ Causado por aranha } \\
\hline Ignorado/Branco & 136.676 & 93,4 \\
\hline Aranha-Armadeira (Phoneutria nigriventer) & 2.869 & 1,9 \\
\hline Aranha-Marrom (Loxosceles spp.) & 1.370 & 0,9 \\
\hline Aranhas-Pretas/Viúvas-Negras (Latrodectus spp.) & 158 & 0,1 \\
\hline Outras espécies & 5.435 & 3,7 \\
\hline
\end{tabular}




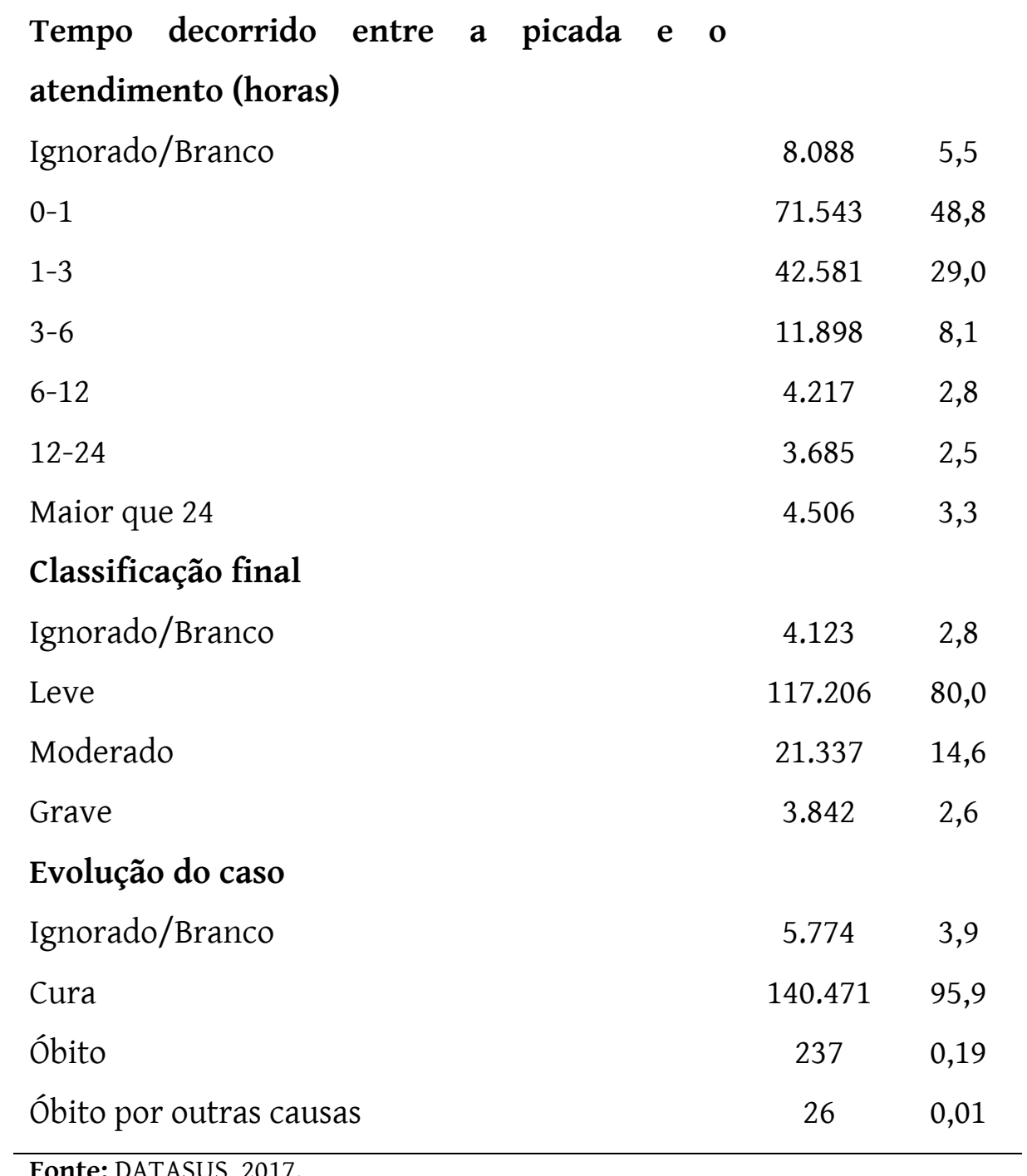

\section{DISCUSSÃO}

Em estudo realizado em Jequié, município baiano, a distribuição dos acidentes de acordo com as estações do ano mostrou uma concentração dos casos durante o outono (35,7\%). Ao se avaliar especificamente os acidentes ofídicos, sua ocorrência foi constatada no decorrer de todo o ano, igualmente com destaque para os meses correspondentes ao outono (36,1\%). Quanto ao escorpionismo, não houve um padrão sazonal, evidenciando-se uma frequência constante durante as estações (CARMO et al., 2016). Neste estudo, os dados do Estado de Minas Gerais corroboram com o estudo realizado em Jequié, Bahia, tendo em vista a prevalência de notificações no primeiro quadrimestre do ano (janeiro-abril), culminando no verão e outono. 
O sexo masculino apresenta uma maior prevalência de vulnerabilidade aos ataques por animais peçonhentos quando comparados às estatísticas femininas. Estudos científicos enfatizam que trabalhadores civis e trabalhadores braçais constantemente tornam-se alvos destes acidentes (OLIVEIRA et al., 2010; LIMA et al., 2009). O ambiente propício destes ataques em mulheres e crianças é a própria residência, porém em menor proporção (LIMA et al., 2009). Em todo o Estado de Minas Gerais, o sexo masculino mantém o alto índice de ataques notificados.

Adultos jovens, com limiar de idade entre 20-40 anos, apresentam melhores condições de recuperação ao tratamento decorrente do pleno funcionamento do sistema imunológico. $\mathrm{O}$ atendimento ambulatorial em tempo hábil torna-se fundamental a fim de se evitar possíveis óbitos advindos da demora do atendimento. Os extremos de idade (crianças e idosos) tendem a ter menor resistência ao veneno quando atingidos. O nível de instrução da pessoa ou da família contribui no surgimento destes agentes causadores, de modo a facilitar o desenvolvimento do ambiente ideal para os mesmos, isso, pois, quanto mais informações o indivíduo apresenta, maior é a prevenção dos fatores de risco ambiental que contribuem para o desenvolvimento ou migração destes agentes causadores (BERTOLOZZI; SCATENA; FRANÇA, 2015). No território mineiro, adultos na faixa etária entre 20-39 anos foram os mais notificados. Vale ressalta que $91,70 \%$ das vítimas não apresentavam dados sobre a escolaridade, de modo a caracterizar uma falha no processo de notificação por parte do responsável pelo atendimento da vítima.

No estudo de Reckziegel (2013), 42,6\% se autodeclararam negras, com maior freqüência de óbitos também nessa raça/cor. Neste estudo, os dados encontrados divergem com os dados da literatura científica, pois aqui é abordado uma maior prevalência de pardos $(42,68 \%)$ e brancos $(33,21 \%)$ acometidos. Os trabalhos científicos já publicados não abordam uma relação intrínseca entre os acidentes e a variável "raça/cor" para explicar os índices estatísticos encontrados.

Em situações cuja mulher é picada por algum animal peçonhento durante a gestação, não há contra-indicação para aplicação do soro em gestantes, devendo as mulheres ter uma atenção especial, pois pode haver descolamento prematuro de placenta e sangramento uterino em decorrência do veneno circular por mais tempo na corrente sanguínea, podendo caracterizar, a depender da idade gestacional (IG), um início de aborto ou um parto 
prematuro (BRASIL, 2003). A maior parte dos notificados não se adéquam à condição gestacional por serem homens $(57,05 \%)$ ou por serem mulheres na qual apresentem quadro clínico que inviabilizem uma gravidez (12,20\%), tal como mulheres histerectomizadas ou em idade avançada. Das mulheres notificadas, prevaleceu a ausência de gestação (23,36\%). E das mulheres que estavam em acompanhamento gestacional, prevaleceram acidentes ocorridos no $2^{\circ}$ trimestre gestacional.

Quanto ao tipo do acidente, boa parte dos trabalhos científicos já publicados retrata o escorpião como precursor das estatísticas de acometimento (AMORIM et al., 2003; LIRADA-SILVA; AMORIM; BRAZIL, 2000; SILVA; CASAIS-E-SILVA; LIRA-DA-SILVA, 2005). Os acidentes ofídicos (CARMO et al., 2016; FISZON; BOCHNER; 2008), seguidos dos acidentes aracnídeos (MARQUES-DA-SILVA; FISCHER, 2005), contemplam grande representatividade estatística das notificações. Os dados do DATASUS convergem com os dados encontrados na bibliografia científica. Dos acidentes ofídicos e aracnídeos já registrados, a espécie causadora do acidente é uma informação pouco registrada ou subnotificada. Neste estudo, o veneno ofídico e aracnídeo com maior prevalência foram, respectivamente, o Botrópico (9,05\%) e o da Aranha-Armadeira (Phoneutria nigriventer) (1,95\%). Outros estudos corroboram estes dados (PINHO; OLIVEIRA; FALEIROS, 2004; BARRELLA; SILVA, 2003; PACHECO et al., 2016).

Após a picada por algum animal venenoso, o tempo hábil entre a mesma e o atendimento ambulatorial torna-se imprescindível. Alguns venenos por serem mais tóxicos que outros, apresentam rápida absorção circulatória e, consequentemente, alta taxa de letalidade. Já outros podem agir mais lentamente no organismo. Sendo assim, quanto menor o tempo na assistência prestada, maior as chances de cura. Os estudos apresentam tempos de atendimento variados, pois este depende dos recursos disponíveis no momento e do ambiente ao qual a vítima e/ou a família se encontram. Oliveira et al. (2010) aborda em seu estudo prevalência de atendimentos realizados em até seis horas após a picada (86,6\% dos pacientes). No estudo de Carmo et al. (2016) feito em Jequié (BA), o tempo de prevalência para internação apresentou intervalo de 1-3 dias, porém o tempo máximo foi de 30 dias. Outro estudo realizado por Lima et al. (2009) na macrorregião de saúde do norte do Estado de Minas Gerais converge com os dados deste estudo de modo a apontar prevalência de atendimento máximo de uma hora após a picada. 
A gravidade do acidente pode ser dada conforme o tempo de procura pelo atendimento, local de acometimento da picada e a presença ou ausência de manifestação sintomatológica. Observa-se que, em Minas Gerais, $80,0 \%$ das notificações foram classificadas como leves e 95,89\% evoluíram para a cura. Uma porcentagem ínfima evoluiu para óbito pelo agravo notificado ( $\mathrm{n}=237 ; 0,19 \%)$. No município de Campina Grande (PB), foram mais frequentes os acidentes classificados como leves $(n=140 ; 70,7 \%)$ causados pelo gênero Bothrops e apenas um paciente evoluiu para óbito (LEMOS et al., 2009). Outros estudos convergem com estes resultados (CARMO et al., 2016; LEITE et al., 2013). Já em outra publicação, casos moderados ou graves prevaleceram (PINHO; OLIVEIRA; FALEIROS, 2004) de modo a divergir com os dados encontrados no presente estudo.

\section{CONCLUSÃO}

$O$ alto índice de ataques por estes animais ocorre devido a causas antrópicas, ou seja, proporcionado pelo próprio homem. Isto é decorrente da migração destes agentes causadores para área comerciais e residenciais devido ao desmatamento e ocupação de seus habitats naturais. Neste estudo, a prevalência de notificações tende a aumentar com o passar dos anos culminando no primeiro trimestre do ano, tendo em vista o período de início das chuvas e do calor com ambiente favorável ao seu desenvolvimento.

Portanto, neste estudo é possível inferir, conforme os achados do estudo, que os adultos jovens do sexo masculino apresentam maior vulnerabilidade aos acidentes por animais peçonhentos, menor predisposição ao desenvolvimento sintomático e maior probabilidade de cura quando atendidos em tempo hábil. 0 escorpião apresenta-se como o principal causador destes acidentes.

\section{REFERÊNCIAS}

AMORIM, A. M.; CARVALHO, F. M.; LIRA-DA-SILVA, R. M.; BRAZIL, T. K. Acidentes por escorpião em uma área do Nordeste de Amaralina, Salvador, Bahia, Brasil. Revista da Sociedade Brasileira de Medicina Tropical. Uberaba, v. 36, n. 1, p. 51-56, 2003. Disponível em: <http://dx.doi.org/10.1590/S0037-86822003000100008>. Acesso em: 30 jul 2017. 
BARRELLA, T. H.; SILVA, R. J. Digenetic trematodes infection in a Bothrops moojeni (Viperidae) population from a fauna rescue in Porto Primavera, São Paulo State. Arquivo Brasileiro de Medicina Veterinária e Zootecnia. Belo Horizonte, v. 55, n. 2, p. 243-245, 2003. Disponível em: <http://dx.doi.org/10.1590/s010209352003000200022>. Acesso em: 30 jul 2017.

BERTOLOZZI, M. R.; SCATENA, C. M. C.; FRANCA, F. O. S. Vulnerabilidades aos acidentes ofídicos em São Paulo. Revista de Saúde Pública. São Paulo, v. 49, Epub 82, p. 1-7, 2015. Disponível em: <http://dx.doi.org/10.1590/S0034-8910.2015049005839>. Acesso em: 30 jul 2017.

BRASIL. Ministério da Saúde. Vigilância Epidemiológica. Fundação Nacional de Saúde. Manual de diagnóstico e tratamento de acidentes por animais peçonhentos. Brasília: FUNASA, 2001. 112p. Disponível em: $<$ http://bvsms.saude.gov.br/bvs/publicacoes/funasa/manu_peconhentos.pdf>. Acesso em: 29 jul 2017.

BRASIL. Ministério da Saúde. Fundação Oswaldo Cruz. Vice-Presidência de Serviços de Referência e Ambiente. Núcleo de Biossegurança. Manual de primeiros socorros. Rio de Janeiro: Fiocruz, 2003. 170p. Disponível em: <http://www.fiocruz.br/biosseguranca/Bis/manuais/biosseguranca/manualdeprimeirossocorros.pdf>. Acesso em: 30 jul 2017.

BRASIL. Ministério da Saúde. Portal Brasil. Ministério da Saúde alerta sobre animais peçonhentos prevenção de acidentes. Brasília, 2014. Disponível em: <http://www.brasil.gov.br/saude/2013/11/ministerioda-saude-alerta-sobre-animais-peconhentos-1>. Acesso em: 29 jul 2017.

BRASIL. Ministério da Saúde. Unidade Técnica de Vigilância de Zoonoses. Sistema de Informação de Agravos de Notificação. Acidente por animais peçonhentos. Brasília: UTVZ/SINAN, 2016. Disponível em: <http://portalsinan.saude.gov.br/acidente-por-animais-peconhentos>. Acesso em: 29 jul 2017.

BRASIL. Ministério da Saúde. Departamento de Informática do Sistema Único de Saúde. Acidente por animais peçonhentos - notificações registradas no sistema de informação de agravos de notificação - Minas Gerais. Brasília: DATASUS, 2017. Disponível em: 〈http://datasus.saude.gov.br/informacoes-de-saude/tabnet>. Acesso em: 29 jul 2017.

CARMO, E. A.; NERY, A. A.; JESUS, C. S.; CASOTTI, C. A. Internações hospitalares por causas externas envolvendo contato com animais em um hospital geral do interior da Bahia, 2009-2011. Epidemiologia \& Serviços de Saúde. Brasília, v. 25, n. 1, p. 105-114, 2016. Disponível em: <http://dx.doi.org/10.5123/s167949742016000100011>. Acesso em: 2017 jul 29.

COSTA, B. C. Fatores de risco para acidentes com escorpiões: uma revisão de literatura. Governador Valadares. 24 fl. Monografia [Especialização em Atenção Básica em Saúde da Família] - Universidade Federal de Minas Gerais, 2011. Disponível em: <https://www.nescon.medicina.ufmg.br/biblioteca/imagem/3096.pdf>. Acesso em: 29 jul 2017.

FISZON, J. T.; BOCHNER, R. Subnotificação de acidentes por animais peçonhentos registrados pelo SINAN no Estado do Rio de Janeiro no período de 2001 a 2005. Revista Brasileira de Epidemiologia. São Paulo, v. 11, n. 1, p. 114-127, 2008. Disponível em: <http://dx.doi.org/10.1590/S1415-790X2008000100011>. Acesso em: 30 jul 2017.

LEITE, R. S.; TARGINO, I. T. G.; LOPES, Y. A. C. F.; BARROS, R. M.; VIEIRA, A. A. Epidemiology of snakebite accidents in the municipalities of the state of Paraíba, Brazil. Ciência \& Saúde Coletiva. Rio de Janeiro, v. 18, n. 5, p. 1463-1471, 2013. Disponível em: <http://dx.doi.org/10.1590/S1413-81232013000500032>. Acessos em: 31 jul 2017.

LEMOS, J. C.; ALMEIDA, T. D.; FOOK, S. M. L.; PAIVA, A. A.; SIMÕES, M. O. S. Epidemiologia dos acidentes ofídicos notificados pelo Centro de Assistência e Informação Toxicológica de Campina Grande (Ceatox-CG), Paraíba. Revista Brasileira de Epidemiologia. São Paulo, v. 12, n. 1, p. 50-59, 2009. Disponível em: <http://dx.doi.org/10.1590/S1415-790X2009000100006>. Acesso em: 31 jul 2017.

LIMA, J. S.; MARTELLI JUNIOR, H.; MARTELLI, D. R. B.; SILVA, M. S.; CARVALHO, S. F. G.; CANELA, J. R.; BONAN, P. R. F. Perfil dos acidentes ofídicos no norte do Estado de Minas Gerais, Brasil. Revista da Sociedade 
Brasileira de Medicina Tropical. Uberaba, v. 42, n. 5, p. 561-564, 2009. Disponível em: <http://dx.doi.org/10.1590/S0037-86822009000500015>. Acesso em: 29 jul 2017.

LIRA-DA-SILVA, R. M.; AMORIM, A. M.; BRAZIL, T. K. Envenenamento por Tityus stigmurus (Scorpiones; Buthidae) no Estado da Bahia, Brasil. Revista da Sociedade Brasileira de Medicina Tropical. Uberaba, v. 33, n. 3, p. 239-245, 2000. Disponível em: <http://dx.doi.org/10.1590/S0037-86822000000300001>. Acesso em: 30 jul 2017.

MARQUES-DA-SILVA, E.; FISCHER, M. L. Distribuição das espécies do gênero Loxosceles Heinecken \& Lowe, 1835 (Araneae; Sicariidae) no Estado do Paraná. Revista da Sociedade Brasileira de Medicina Tropical. Uberaba, v. 38, n. 4, p. 331-335, 2005. Disponível em: <http://dx.doi.org/10.1590/S0037-86822005000400010>. Acesso em: 30 jul 2017.

OLIVEIRA, F. N.; BRITO, M. T.; MORAIS, I. C. O.; FOOK, S. M. L.; ALBUQUERQUE, H. N. Accidents caused by Bothrops and Bothropoides in the State of Paraiba: epidemiological and clinical aspects. Revista da Sociedade Brasileira de Medicina Tropical. Uberaba, v. 43, n. 6, p. 662-667, 2010. Disponível em: <http://dx.doi.org/10.1590/S0037-86822010000600012>. Acesso em: 29 jul 2017.

PACHECO, D. F.; FREITAS, ACN; PIMENTA, A. M. C.; DUARTE, I. D. G.; LIMA, M. E. A spider derived peptide, PnPP19 , induces central antinociception mediated by opioid and cannabinoid systems. Journal of Venomous Animals and Toxins including Tropical Diseases. Botucatu, v. 22, Epub 34, p. 1-7, 2016. Disponível em: <http://dx.doi.org/10.1186/s40409-016-0091-6>. Acesso em: 30 jul 2017.

PINHO, F. M. O.; OLIVEIRA, E. S.; FALEIROS, F. Acidente ofídico no estado de Goiás. Revista da Associação Médica Brasileira. São Paulo, v. 50, n. 1, p. 93-96, 2004. Disponível em: <http://dx.doi.org/10.1590/S010442302004000100043>. Acesso em: 30 jul 2017.

RECKZIEGEL, G. C. Análise do escorpionismo no Brasil no período de 2000 a 2010. Brasília. 103 fl. Dissertação [Mestrado em Saúde Coletiva] - Faculdade de Ciências da Saúde, Universidade de Brasília, 2013. Disponível

em: <http://repositorio.unb.br/bitstream/10482/14169/5/2013_\%20GuilhermeCarneiroReckziegel.pdf>. Acesso em: 30 jul 2017.

SANTANA, V. T. P.; SUCHARA, E. A. Epidemiologia dos acidentes com animais peçonhentos registrados em Nova Xavantina - MT. Revista de Epidemiologia e Controle de Infecção. Santa Cruz do Sul, v. 5, n. 3, p. 141146, $2015 . \quad$ Disponível em: <https://online.unisc.br/seer/index.php/epidemiologia/article/download/5724/4461>. Acesso em: 29 jul 2017.

SILVA, T. F.; CASAIS-E-SILVA, L. L.; LIRA-DA-SILVA, R. M. Avaliação da DL $_{50}$ e edema pulmonar induzido pelo veneno de Tityus serrulatus (Scorpiones; Buthidae) procedente da Bahia, Brasil. Biota Neotrópica. Campinas, v. 5, n. 1, p. 221-224, 2005. Disponível em: <http://dx.doi.org/10.1590/S1676-06032005000200023>. Acesso em: 30 jul 2017.

SILVA, S. T.; TIBURCIO, I. C. S.; CORREIA, G. Q. C.; AQUINO, R. C. T. Escorpiões, aranhas e serpentes: aspectos gerais e espécies de interesse médico no Estado de Alagoas. Maceió: Editora UFAL, 2005. 54p. (Série: Conversando sobre ciências em Alagoas). Disponível em: <http://www.usinaciencia.ufal.br/multimidia/livrosdigitais-cadernos-tematicos/Escorpioes_Aranhas_e_Serpentes.pdf>. Acesso em: 29 jul 2017. 